\title{
Caracterización físico-química del sistema bentónico en el sector sur del Golfo San Jorge, Argentina
}

\author{
Physico-chemical characterization of the benthic system in the south area of \\ the San Jorge Gulf, Argentina
}

\author{
Mónica Fernández ${ }^{1}$, Daniel Cucchi Colleoni ${ }^{1}$, Ana Roux ${ }^{1}$, Ángel Marcos ${ }^{2}$ y Elba Fernández ${ }^{2}$ \\ ${ }^{1}$ Instituto Nacional de Investigación y Desarrollo Pesquero (INIDEP) Paseo Victoria Ocampo 1, B7602HSA, \\ Mar del Plata, Argentina \\ ${ }^{2}$ Universidad Nacional del Sur, Departamento de Geología San Juan 670, 8000, Bahía Blanca, Argentina \\ monica-fernandez@inidep.edu.ar
}

\begin{abstract}
Surficial sediments and bottom water physicochemical characteristics of the San Jorge Gulf are presented corresponding to the main concentration area of the shrimp Pleoticus muelleri (Bate, 1888) which sustents the fishery of the patagonic littoral.

Data were obtained during the research cruise (OB-05/03) on board the RV Capitán Oca Balda of the INIDEP, during June 2003. Environmental variables such as temperature, salinity, density, oxygen and bottom water chlorophyll $a$ content and granulometric fractions, total organic matter, total organic carbon, total nitrogen, total phosphorous, chlorophyll $a$ and phaeopigments in sediment concentration as well as depth were analysed. The origin and nutritional value of the deposited organic matter were also assessed.

Cluster analysis was applied for the numeric treatment of the data. Spatial distribution of the variables defined two sectors in the south area of the San Jorge Gulf, corresponding to the Depositional Environment (low hydrographic conditions, fine granulometric fractions and high concentration of organic material in sediments) and the Transitional Environment (intermediate hydrodynamic conditions, medium granulometric fractions and moderate organic material concentration in sediments), both previously described by Fernández et al. (2003, 2005, 2006).

This research pretends a physico-chemical characterization of the environment where the highest concentrations of the fishery resource are located. This will allow a posterior analysis related to the distribution patterns and abundance of the shrimp in the south sector of the San Jorge Gulf, due to the relationship noticed among the abundance index of the species and some of the oceanographic and sediment variables.
\end{abstract}

Key words: Benthic environment, sediments, bottom water, shrimp fishery, Patagonia
Resumen.- Se presenta la caracterización físico-química de los sedimentos superficiales y del agua de fondo del área sur del Golfo San Jorge, zona donde se localizan las mayores concentraciones de langostinos que sustentan la pesquería del litoral patagónico. Los datos analizados provienen de la Campaña de Investigación OB-05/03 realizada en junio de 2003 con el BIP Capitán Oca Balda del INIDEP. Se analizaron las variables ambientales: temperatura, salinidad, densidad, contenido de oxígeno y de clorofila $a$ del agua de fondo y fracciones granulométricas, concentración de materia orgánica total, carbono orgánico total, nitrógeno total, fósforo total, clorofila $a$ y feopigmentos en sedimentos, como así también, profundidad. El origen y el valor nutricional de la materia orgánica sedimentada también fueron contemplados. Para el tratamiento numérico de los datos se aplicó la técnica de clasificación. La distribución espacial de las variables coincidió con la definición de dos sectores en el área sur del Golfo San Jorge, correspondientes al Ambiente Deposicional (de condiciones hidrodinámicas bajas, depositación de elementos granulométricos finos y abundante material orgánico en sedimentos) y al Ambiente Transicional (de condiciones hidrodinámicas medias, granulometría mediana y concentraciones moderadas de material orgánico en sedimentos), ambos previamente descritos por Fernández et al. $(2003,2005,2006)$.

Esta investigación está orientada a caracterizar desde un punto de vista físico-químico el ambiente donde se registran las mayores concentraciones de este recurso pesquero, para un posterior análisis relacionado con los patrones de distribución y abundancia del langostino, en el sector sur del Golfo San Jorge, dado que se advierte una relación entre los índices de abundancia de la especie y algunas de las variables oceanográficas y sedimentológicas.

Palabras clave: Ambiente bentónico, sedimentos, agua de fondo, pesquería de langostino, Patagonia

Contribución del INIDEP N 1414 


\section{Introducción}

El Golfo San Jorge es una cuenca semiabierta del Mar Argentino ubicada aproximadamente entre la latitud $45^{\circ}$ y $47^{\circ} \mathrm{S}$ (desde el cabo Dos Bahías hasta el cabo Tres Puntas) y el meridiano $65^{\circ} 30^{\prime} \mathrm{W}$ y la costa, que ocupa una superficie de $39.340 \mathrm{~km}^{2}$. Las mayores profundidades del golfo $(<110 \mathrm{~m})$ se registran en la región central (Fernández et al. 2005).

Las aguas del golfo pertenecen a la denominada agua de plataforma, modificada por los aportes de aguas costeras, provenientes del Estrecho de Magallanes. En los extremos del golfo, de menor profundidad, se generan frentes de marea de desarrollo estacional y permanencia durante la primavera y fines del otoño. Además, las aguas costeras generan en su extremo sur un intenso frente termohalino de permanencia estacional y de posición variable (Fernández et al. 2005).

Este golfo patagónico es de gran importancia económica por ser caladero de especies de interés comercial entre las que se destacan el langostino Pleoticus muelleri (Bertuche et al. 2000) y la merluza común Merluccius hubbsi (Aubone et al. 2000).

El área sur, comprendida entre las latitudes $46^{\circ} 30^{\prime} \mathrm{S}$ y $47^{\circ} 00^{\prime} \mathrm{S}$ y el meridiano $66^{\circ} 00^{\prime} \mathrm{W}$ y la costa, es un sector de particular importancia en relación al recurso langostino ya que en el mismo se localizan las mayores concentraciones que sustentan a la pesquería del litoral patagónico.

Dada la vinculación existente entre el sistema bentónico y el recurso langostino en las diferentes etapas de su ciclo de vida ya sea por alimentación y/o refugio, se iniciaron a partir de 1992 (Fernández \& Bertuche 1995) diversos estudios orientados al conocimiento de los factores físico-químicos del agua, de los sedimentos superficiales así como de las comunidades bentónicas en los fondos de distribución del langostino (Roux et al. 1995, Roux \& Fernández 1997).

Estas líneas de investigación también han sido desarrolladas en diversas pesquerías de peneidos (Grady, Loesch 1965, Branford 1981, Somers 1987).
El presente estudio caracteriza el área sur del Golfo San Jorge a través de variables físico-químicas con el objeto de relacionar en futuras investigaciones los patrones de distribución y abundancia del langostino con las variables estudiadas.

\section{Materiales y métodos}

Los datos y las muestras provienen de la Campaña de Investigación del BIP Capitán Oca Balda del INIDEP, OB-05/03, llevada a cabo en otoño-invierno de 2003 (9 al 29 de junio) en el Golfo San Jorge, Argentina. En la Fig. 1 se representa la ubicación de las estaciones generales de muestreo y la localización de la transección seleccionada para la representación de los perfiles verticales de temperatura y salinidad.

En la columna de agua, las mediciones de presión, temperatura y conductividad se realizaron con un CTD SBE (Sea-Bird Electronic) modelo XIX, con fluorómetro (Seapoint Chlorophyll Fluorometer) adosado para la determinación "in situ" de los perfiles verticales de fluorescencia del fitoplancton "in vivo". La toma de muestras para los análisis hidrológicos (determinación de oxígeno disuelto y clorofila $a$ ) se realizó con ayuda de botellas tipo Niskin, tomándose muestras de agua de fondo.

Las muestras de sedimentos fueron obtenidas con el extractor de gravedad Phleger (3 réplicas) y rastra tipo Picard (2 réplicas) y conservadas a bordo a $-25{ }^{\circ} \mathrm{C}$ para su posterior análisis en el laboratorio.

Las determinaciones de oxígeno disuelto $(\mathrm{O})$ se realizaron mediante el método de Winkler (Yodometría) y las de clorofila $a$ en agua de fondo $(\mathrm{Cl}$ $a(\mathrm{AF}))$ a través de la técnica de espectrofluorometría (Strickland \& Parsons 1965).

El análisis granulométrico de los sedimentos se realizó siguiendo el método convencional descripto por Buchanan (1984).

Las determinaciones de materia orgánica total (MOT), carbono orgánico total (COT), nitrógeno total (NT), fósforo total (FT), valor nutricional y origen de la materia orgánica (relación C:N), clorofila $a(\mathrm{Cl} a(\mathrm{~S}))$ y feopigmentos (Feop (S)) en sedimentos, se realizó aplicando la metodología claramente descripta en Fernández (2006). 


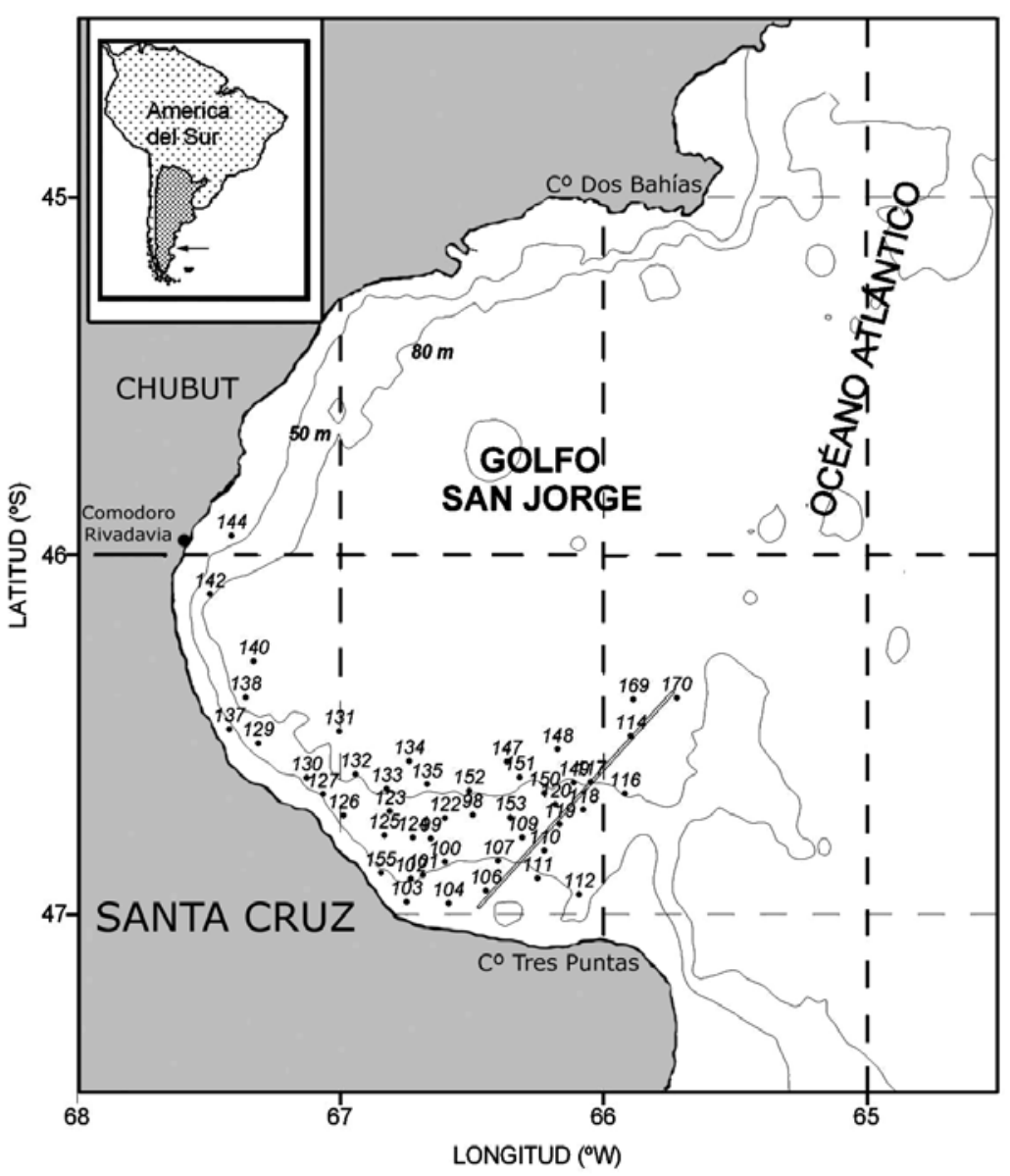

Figura 1

Ubicación de las estaciones generales de muestreo y localización de la transección seleccionada para la representación de los perfiles verticales de temperatura y salinidad en el sector sur del Golfo San Jorge

Geographical localization of sampling stations and the selected transect for temperature and salinity vertical profiles in the south sector of the San Jorge Gulf

Para clasificar y ordenar las 46 estaciones de muestreo y las 27 variables: profundidad (P), 15 clases texturales, temperatura $(\mathrm{T})$, salinidad $(\mathrm{S})$, oxígeno disuelto $(\mathrm{O})$ y clorofila $a(\mathrm{Cl}$ a $(\mathrm{AF}))$ en agua de fondo, MOT, COT, NT, C:N, FT, Cl a (S) y feop. (S) en sedimentos, se aplicó la técnica de agrupamiento, previa elaboración de la matriz correspondiente con datos estandarizados (Fernández et al. 2003, 2005, 2006). El análisis estadístico se realizó usando el programa Statistica 1998. La representación cartográfica de los diferentes factores físico-químicos se elaboró mediante el programa gráfico Surfer versión 7 (Golden Software) empleando para la interpolación el método de "kriging".

\section{Resultados}

\section{Variables hidrográficas}

\section{Temperatura ( $T$ )}

El rango de variación de la temperatura del agua de fondo fue de $8,39{ }^{\circ} \mathrm{C}$ a $9,76{ }^{\circ} \mathrm{C}$ (Tablas 1 y 2). Los valores máximos fueron registrados en la zona costera somera y los mínimos hacia el sector central (Fig. 2). En el sector costero sur, las isotermas se bifurcaron en una rama costera oeste y otra hacia el océano. La distribución vertical de la temperatura en la columna de agua fue prácticamente homogénea entre superficie y fondo (Fig. 2). 


\section{Salinidad (S)}

La salinidad determinada en el área, estuvo comprendida entre 33,134 a 33,558 ups (Tablas 1 y 2). La distribución horizontal de la salinidad sobre el fondo (Fig. 2) siguió un patrón caracterizado por: 1) bajos valores en el sector costero sur con mínimos en el cuadrante SE (33,134 ups), 2) una prolongación de baja salinidad en sentido norte por el exterior del golfo en forma de lengua, 3 ) un incremento rápido de isohalinas desde el sector sur hacia el área central con valores de hasta 33,558 ups y 4) un incremento de isohalinas desde el sector sur hacia el área costera oeste con

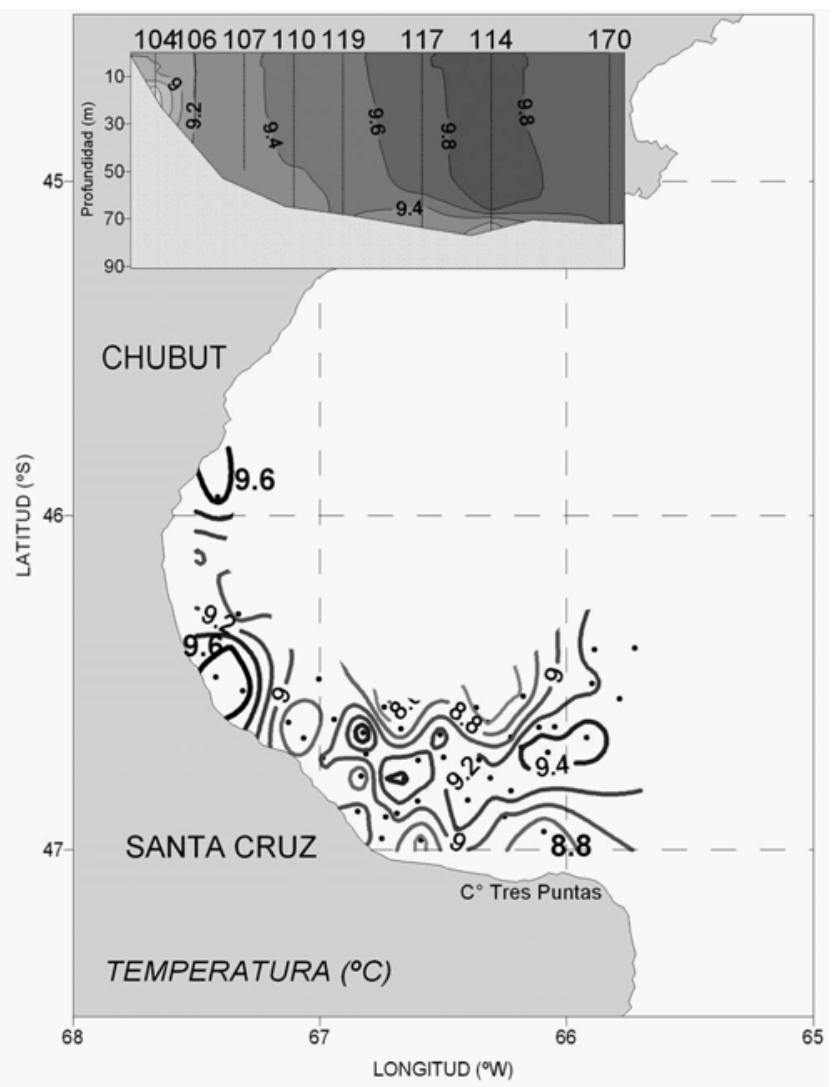

valores de hasta 33,501 ups. La distribución de esta variable en la columna de agua denota ausencia de haloclina y una disminución de la salinidad desde la costa hacia el sector central del umbral del golfo con valores inferiores a 33,30 ups.

\section{Densidad (D)}

La distribución horizontal sobre el fondo de la densidad fluctuó entre 25,674 y 26,084 (Tablas 1 y 2). Las isolíneas indicaron un patrón caracterizado por presentar valores crecientes desde el cuadrante SE hacia el interior y litoral costero oeste del golfo.

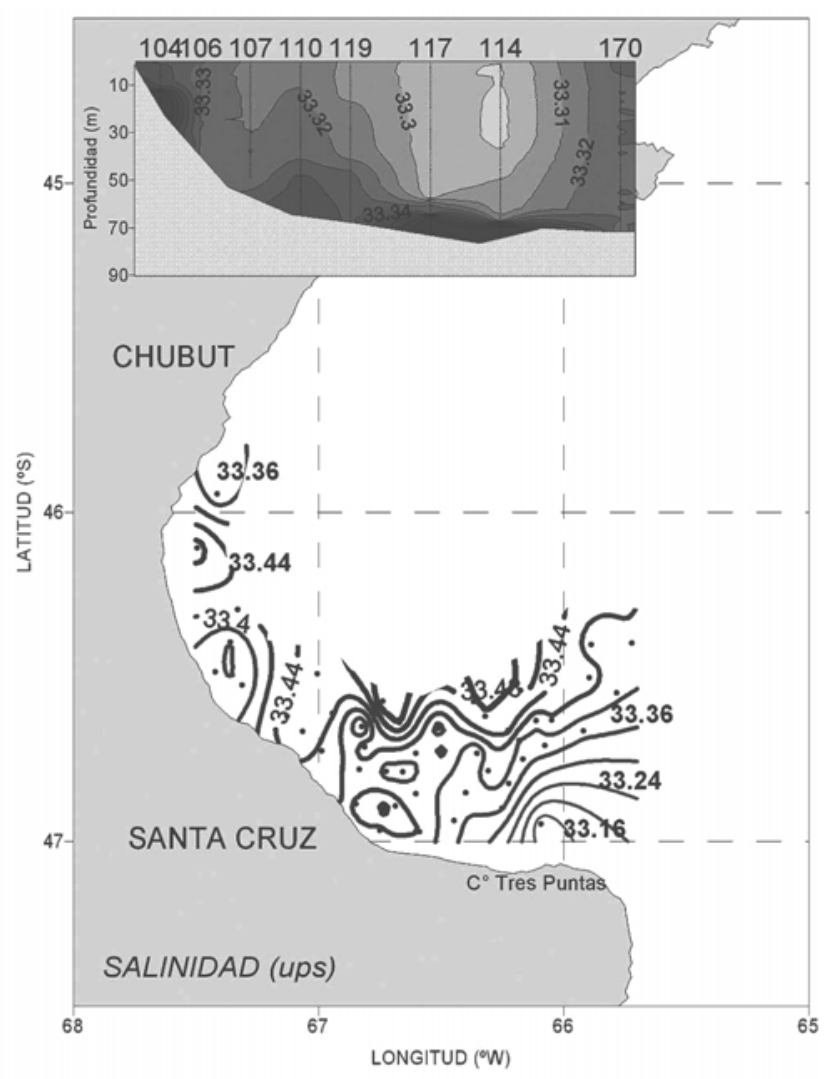

Figura 2

Distribución horizontal de la temperatura $\left({ }^{\circ} \mathrm{C}\right.$ ) y salinidad (ups) del agua de fondo y perfiles verticales de la columna de agua en el sector sur del Golfo San Jorge

Horizontal distribution of bottom water temperature $\left({ }^{\circ} \mathrm{C}\right)$ and salinity $(\mathrm{psu})$ and vertical profiles of the water column in the south sector of the San Jorge Gulf 
Tabla 1

Estadística descriptiva de las variables físico-químicas analizadas en el agua de fondo y en el sedimento superficial del sector sur del Golfo San Jorge

Descriptive statistic of the physico-chemical variables analysed in bottom water and surficial sediment in the south sector of the San Jorge Gulf

\begin{tabular}{lcccc}
\hline \multicolumn{1}{c}{ Variables } & $\begin{array}{c}\text { Valor } \\
\text { mínimo }\end{array}$ & $\begin{array}{c}\text { Valor } \\
\text { máximo }\end{array}$ & $\begin{array}{c}\text { Valor } \\
\text { promedio }\end{array}$ & $\begin{array}{c}\text { Desviación } \\
\text { estándar }\end{array}$ \\
\hline Profundidad (m) & 31,00 & 91,00 & 69,76 & 14,84 \\
Arena muy gruesa (\%) & 44,95 & 44,95 & 44,95 & - \\
Arena gruesa (\%) & 3,04 & 3,04 & 3,04 & - \\
Arena gruesa (\%) & 2,39 & 2,39 & 2,39 & - \\
Total arena gruesa a muy gruesa (\%) & 50,38 & 50,38 & 50,38 & - \\
Arena mediana (\%) & 3,26 & 3,26 & 3,26 & - \\
Arena mediana (\%) & 2,94 & 10,1 & 6,52 & 5,06 \\
Total arena mediana (\%) & 2,94 & 13,36 & 8,15 & 7,37 \\
Arena fina (\%) & 0 & 12,05 & 0,53 & 1,92 \\
Arena fina (\%) & 0 & 48,89 & 4,12 & 9,32 \\
Total arena fina (\%) & 0 & 51,92 & 4,64 & 10,04 \\
Arena muy fina (\%) & 0 & 40,29 & 9,64 & 13,08 \\
TOTAL ARENA (\%) & 0 & 79,16 & 15,73 & 21,70 \\
Limo gruesa (\%) & 0,92 & 47,31 & 13,50 & 9,03 \\
Limo medio (\%) & 0,03 & 19,59 & 6,51 & 4,72 \\
Limo fino (\%) & 1,19 & 31,65 & 10,30 & 5,80 \\
Limo muy fino (\%) & 2,1 & 20,29 & 11,03 & 5,39 \\
TOTAL LIMO (\%) & 9,68 & 65,93 & 41,34 & 12,20 \\
Arcilla gruesa (\%) & 0,87 & 61,25 & 16,71 & 13,92 \\
Arcilla mediana (\%) & 0,97 & 18,35 & 8,33 & 4,56 \\
Arcilla fina a muy fina (\%) & 2 & 58,575 & 17,89 & 11,94 \\
TOTAL ARCILLA (\%) & 10,15 & 73,875 & 42,93 & 18,85 \\
Materia orgánica total $(\%)$ & 0,78 & 11,85 & 6,56 & 2,83 \\
Carbono orgánico total $(\%)$ & 0,34 & 1,53 & 0,97 & 0,35 \\
Nitrógeno total (\%) & 0,05 & 0,27 & 0,15 & 0,06 \\
Fósforo total (\%) & 0,03 & 0,09 & 0,05 & 0,01 \\
Relación Carbono:Nitrógeno & 4,73 & 8,54 & 6,68 & 1,07 \\
Clorofila $a\left(\right.$ Sedimentos) $\left(\mu \mathrm{g} \mathrm{g}^{-1}\right)$ & 0,20 & 9,90 & 1,31 & 1,45 \\
Feopigmentos (Sedimentos) $\left(\mu \mathrm{g} \mathrm{g}^{-1}\right)$ & 1,74 & 43,19 & 11,98 & 8,04 \\
Temperatura ( $\left.{ }^{-1} \mathrm{C}\right)$ & 8,39 & 9,76 & 9,10 & 0,36 \\
Salinidad (ups) & 33,134 & 33,558 & 33,390 & 0,08 \\
Densidad ( $\sigma_{\mathrm{t}}$ ) & 25,674 & 26,084 & 25,848 & 0,109 \\
Concentración de Oxígeno $\left(\mathrm{O}_{2} \mathrm{~L}^{-1}\right)$ & 1,30 & 6,27 & 4,81 & 1,17 \\
Clorofila $a\left(\right.$ Agua de fondo) $\left(\mathrm{mg} \mathrm{m}^{-3}\right)$ & 0,02 & 0,91 & 0,43 & 0,25 \\
\hline & & & & \\
\hline
\end{tabular}


Tabla 2

Profundidad (P), temperatura (T), salinidad (S), densidad (D), concentración de oxígeno (O) y clorofila a en agua de fondo (Cl a AF); materia orgánica total (MOT), carbono orgánico total (COT), nitrógeno total (NT), fósforo total (FT), relación carbono-nitrógeno (C:N), clorofila $a(\mathrm{Cl}$ a S) y feopigmentos (Feop S) en sedimentos, de cada estación de muestreo en el sector sur del Golfo San Jorge

Depth (D), temperature (T), salinity (S), density (D), oxygen concentration (O) and bottom water chlorophyll $a(\mathrm{Cl} a \mathrm{BW})$; total organic matter (TOC), total organic carbon (TOC), total nitrogen (TN), total phosphorous (TP), Carbon-Nitrogen ratio (C:N), chlorophyll $a(\mathrm{Cl} a \mathrm{~S})$ and phaeopigments (Phaeo S) in sediments of each sampling station in the south sector of the San Jorge Gulf

\begin{tabular}{|c|c|c|c|c|c|c|c|c|c|c|c|c|c|}
\hline $\begin{array}{c}\text { Estación } \\
\text { General } \\
\end{array}$ & $\begin{array}{l}\mathbf{P} \\
\mathrm{m}\end{array}$ & $\begin{array}{c}\mathrm{T} \\
{ }^{\circ} \mathrm{C} \\
\end{array}$ & $\begin{array}{c}\mathbf{S} \\
\text { ups }\end{array}$ & $\begin{array}{l}\mathbf{D} \\
\sigma_{\mathrm{t}} \\
\end{array}$ & $\begin{array}{c}\mathbf{O} \\
\mathrm{O}_{2} \mathrm{~L}^{-1} \\
\end{array}$ & $\begin{array}{c}\mathrm{Cl} \boldsymbol{a} \text { (AF) } \\
\mathrm{mg} \mathrm{m}^{-3}\end{array}$ & $\begin{array}{c}\text { MOT } \\
\% \\
\end{array}$ & $\begin{array}{c}\text { COT } \\
\% \\
\end{array}$ & $\begin{array}{c}\text { NT } \\
\% \\
\end{array}$ & $\begin{array}{c}\text { FT } \\
\% \\
\end{array}$ & $C: N$ & $\begin{array}{c}\text { Cla (S) } \\
\mu \mathrm{g} \mathrm{g}^{-1}\end{array}$ & $\begin{array}{c}\text { feop (S) } \\
\mu \mathrm{g} \mathrm{g}^{-1}\end{array}$ \\
\hline 98 & 68 & 9,07 & 33,415 & 25,867 & 4,45 & 0,38 & 6,58 & 1,16 & 0,16 & 0,049 & 7,09 & 1,62 & 21,76 \\
\hline 99 & 58 & 9,44 & 33,346 & 25,757 & 5,48 & 0,60 & 7,32 & 1,31 & 0,20 & 0,043 & 6,56 & 1,06 & 13,02 \\
\hline 100 & 53 & 9,07 & 33,374 & 25,841 & 5,16 & 0,60 & 6,64 & 1,15 & 0,17 & 0,046 & 6,94 & 1,70 & 4,53 \\
\hline 101 & 51 & 9,13 & 33,417 & 25,874 & 4,02 & 0,47 & 10,65 & 1,06 & 0,19 & 0,042 & 8,39 & 0,76 & 9,41 \\
\hline 102 & 58 & 8,82 & 33,459 & 25,949 & 3,41 & 0,30 & 6,92 & 1,08 & 0,18 & 0,046 & 7,59 & 0,70 & 11,87 \\
\hline 104 & 31 & 8,51 & 33,401 & 25,942 & 5,52 & 0,77 & 2,91 & 0,34 & 0,05 & 0,039 & 6,37 & 0,56 & 3,05 \\
\hline 106 & 31 & 9,22 & 33,330 & 25,775 & 5,58 & 0,75 & 3,05 & 0,35 & 0,07 & 0,035 & 5,40 & 0,67 & 2,57 \\
\hline 107 & 49 & 9,33 & 33,324 & 25,753 & 5,56 & 0,91 & 4,04 & 0,89 & 0,11 & 0,039 & 7,78 & 1,91 & 8,34 \\
\hline 109 & 70 & 9,20 & 33,394 & 25,830 & 4,56 & 0,49 & 4,48 & 0,79 & 0,13 & 0,043 & 6,28 & 1,07 & 7,14 \\
\hline 110 & 74 & 9,37 & 33,348 & 25,766 & 5,18 & 0,74 & 4,21 & 0,73 & 0,10 & 0,041 & 7,48 & 1,14 & 7,46 \\
\hline 111 & 48 & 8,97 & 33,326 & 25,735 & 5,17 & 0,56 & 4,30 & 0,58 & 0,07 & 0,035 & 8,39 & 0,54 & 4,44 \\
\hline 112 & 55 & 8,60 & 33,134 & 25,720 & 6,27 & 0,70 & 2,01 & 0,51 & 0,06 & 0,036 & 8,26 & 1,07 & 1,74 \\
\hline 114 & 86 & 9,13 & 33,422 & 25,864 & 3,81 & 0,25 & 3,59 & 0,62 & 0,08 & 0,048 & 7,85 & 0,50 & 6,13 \\
\hline 116 & 77 & 9,52 & 33,333 & 25,727 & 5,12 & 0,51 & 3,49 & 0,66 & 0,08 & 0,042 & 8,51 & 1,77 & 6,66 \\
\hline 117 & 84 & 9,29 & 33,395 & 25,816 & 4,26 & 0,32 & 3,87 & 0,95 & 0,13 & 0,047 & 7,55 & 0,33 & 4,60 \\
\hline 118 & 76 & 9,49 & 33,345 & 25,745 & 4,98 & 0,15 & 3,43 & 0,84 & 0,11 & 0,047 & 7,47 & 1,10 & 8,48 \\
\hline 119 & 76 & 9,40 & 33,341 & 25,756 & 5,19 & 0,25 & 2,99 & 0,50 & 0,06 & 0,043 & 8,40 & 0,58 & 4,17 \\
\hline 120 & 76 & 9,42 & 33,357 & 25,766 & 4,96 & 0,28 & 4,21 & 0,83 & 0,11 & 0,049 & 7,71 & 0,98 & 8,70 \\
\hline 122 & 66 & 9,29 & 33,365 & 25,827 & 4,85 & 0,25 & 9,13 & 1,24 & 0,20 & 0,060 & 6,08 & 2,39 & 16,43 \\
\hline 123 & 73 & 9,12 & 33,426 & 25,674 & 5,14 & 0,46 & 10,65 & 1,26 & 0,20 & 0,058 & 6,90 & 1,24 & 18,29 \\
\hline 124 & 64 & 9,42 & 33,342 & 25,754 & 5,26 & 0,36 & 9,73 & 1,37 & 0,21 & 0,057 & 6,52 & 1,85 & 14,65 \\
\hline 125 & 65 & 8,59 & 33,385 & 25,917 & 5,38 & 0,44 & 9,35 & 1,33 & 0,20 & 0,057 & 6,63 & 0,65 & 12,22 \\
\hline 126 & 56 & 9,03 & 33,431 & 25,891 & 3,89 & 0,29 & 10,48 & 1,13 & 0,17 & 0,052 & 6,62 & 1,21 & 15,72 \\
\hline 127 & 71 & 8,65 & 33,477 & 25,985 & 3,05 & 0,19 & 7,63 & 1,10 & 0,17 & 0,054 & 6,61 & 0,87 & 14,31 \\
\hline 129 & 71 & 9,76 & 33,324 & 25,692 & 5,82 & 0,79 & 9,12 & 1,32 & 0,24 & 0,054 & 5,62 & 0,75 & 14,50 \\
\hline 130 & 64 & 8,67 & 33,471 & 25,976 & 3,62 & 0,24 & 8,39 & 1,13 & 0,19 & 0,053 & 6,12 & 0,90 & 18,36 \\
\hline 133 & 79 & 9,65 & 33,309 & 25,872 & 6,20 & 0,24 & 9,96 & 1,40 & 0,21 & 0,068 & 6,52 & 1,34 & 17,64 \\
\hline 134 & 87 & 8,39 & 33,558 & 26,084 & 2,13 & 0,02 & 9,46 & 1,43 & 0,22 & 0,056 & 6,56 & 0,65 & 15,08 \\
\hline 135 & 85 & 8,57 & 33,526 & 26,038 & 5,73 & 0,48 & 9,16 & 1,38 & 0,26 & 0,064 & 5,27 & 0,49 & 9,88 \\
\hline 138 & 80 & 9,63 & 33,317 & 25,724 & 5,85 & 0,65 & 8,56 & 1,50 & 0,20 & 0,054 & 8,54 & 1,19 & 11,93 \\
\hline 140 & 80 & 8,87 & 33,423 & 26,066 & 5,72 & 0,05 & 9,38 & 1,46 & 0,26 & 0,054 & 5,67 & 9,90 & 43,19 \\
\hline 142 & 81 & 8,97 & 33,501 & 25,980 & 1,30 & 0,03 & 8,93 & 1,53 & 0,27 & 0,089 & 6,08 & 0,52 & 8,45 \\
\hline 144 & 66 & 9,68 & 33,329 & 25,724 & 5,70 & 0,63 & 2,91 & 0,44 & 0,09 & 0,071 & 4,73 & 0,21 & 2,62 \\
\hline 147 & 89 & 8,55 & 33,515 & 26,014 & 2,74 & 0,14 & 8,27 & 0,79 & 0,14 & 0,056 & 5,63 & 2,01 & 19,81 \\
\hline 148 & 86 & 8,62 & 33,510 & 26,014 & 2,90 & 0,03 & 5,30 & 0,60 & 0,11 & 0,056 & 5,46 & 0,74 & 5,23 \\
\hline 149 & 76 & 9,34 & 33,366 & 25,784 & 5,88 & 0,74 & 5,35 & 0,47 & 0,10 & 0,049 & 4,94 & 1,48 & 11,72 \\
\hline 150 & 73 & 8,93 & 33,449 & 25,924 & 3,81 & 0,17 & 4,38 & 0,60 & 0,11 & 0,056 & 5,39 & 0,67 & 10,96 \\
\hline 151 & 82 & 8,57 & 33,514 & 26,021 & 2,95 & 0,53 & 6,34 & 0,77 & 0,14 & 0,056 & 5,46 & 1,14 & 14,29 \\
\hline 152 & 81 & 9,32 & 33,336 & 25,766 & 5,64 & 0,12 & 8,49 & 1,17 & 0,20 & 0,067 & 5,81 & 1,75 & 27,39 \\
\hline 153 & 76 & 9,28 & 33,318 & 25,757 & 5,86 & 0,68 & 6,49 & 0,88 & 0,14 & 0,053 & 6,36 & 2,62 & 12,86 \\
\hline 155 & 56 & 9,10 & 33,401 & 25,853 & 4,68 & 0,43 & 8,94 & 0,87 & 0,11 & 0,047 & 7,94 & 1,27 & 11,91 \\
\hline 169 & 91 & 9,34 & 33,377 & 25,857 & 6,08 & 0,46 & 4,14 & 0,50 & 0,08 & 0,046 & 6,25 & 0,20 & 5,06 \\
\hline 170 & 91 & 9,30 & 33,399 & 25,819 & 6,09 & 0,71 & 4,93 & 0,65 & 0,14 & 0,051 & 4,81 & 0,21 & 3,62 \\
\hline
\end{tabular}




\section{Concentración de oxígeno disuelto (O)}

El oxígeno disuelto fluctuó entre $1,30 \mathrm{~mL} \mathrm{O}_{2} \mathrm{~L}^{-1}$ y 6,27 $\mathrm{mL} \mathrm{O}_{2} \mathrm{~L}^{-1}$ (Tablas 1 y 2). La distribución horizontal sobre el fondo de la concentración de oxígeno disuelto presenta valores máximos en los sectores costeros (entre $5,72 \mathrm{~mL} \mathrm{O}_{2} \mathrm{~L}^{-1}$ a $6,27 \mathrm{~mL} \mathrm{O}_{2} \mathrm{~L}^{-1}$ ) y mínimos en el sector central del golfo (entre $2,95 \mathrm{~mL} \mathrm{O}_{2} \mathrm{~L}^{-1}$ y 2,13 $\mathrm{mL} \mathrm{O}_{2} \mathrm{~L}^{-1}$ ).

\section{Concentración de clorofila a (Cl $a$ (AF))}

La concentración de clorofila $a$ (Tablas 1 y 2) varió entre $0,02 \mathrm{mg} \mathrm{m}^{-3}$ y $0,91 \mathrm{mg} \mathrm{m}^{-3}$ con un valor medio de $0,43 \mathrm{mg} \mathrm{m}^{-3}$. La distribución horizontal sobre el fondo de esta variable, muestra un patrón de: 1) valores altos en el sector costero sur $\left(0,65 \mathrm{mg} \mathrm{m}^{-3}\right.$ a $\left.0,91 \mathrm{mg} \mathrm{m}^{-3}\right) \mathrm{y}$ en el cuadrante SE $\left(0,56 \mathrm{mg} \mathrm{m}^{-3}\right.$ a $\left.0,71 \mathrm{mg} \mathrm{m}^{-3}\right)$ y 2$)$ una disminución de los valores hacia el interior del golfo con valores entre $0,02 \mathrm{mg} \mathrm{m}^{-3}$ y $0,15 \mathrm{mg} \mathrm{m}^{-3}$ y hacia el sector costero oeste, con registro de valores mínimos entre 0,02 y $0,05 \mathrm{mg} \mathrm{m}^{-3}$.

\section{Variables sedimentológicas}

\section{Caracterización física del sedimento}

Los resultados de los análisis granulométricos realizados en los sedimentos superficiales del sector sur del Golfo San Jorge (Tabla 3 y Fig. 3), indican 15 clases de grano, con un rango de tamaño de grano entre arena muy gruesa $(\Phi=0$ o grano de $1 \mathrm{~mm})$ y arcilla muy fina $(\Phi<0,001 \mathrm{~mm})$. Considerando la composición porcentual en peso de las clases texturales arena, limo y arcilla, la mayor participación se concentró en la clase arcilla.

La fracción arena (Fig. 3) se localizó en el sector sudeste del golfo y frente a la ciudad de Comodoro Rivadavia y representó el $6 \%$ de las estaciones analizadas. La fracción fango (limo y arcilla) (Fig. 3) predominó en el resto de la zona relevada, representando el $94 \%$ de las estaciones. El $46 \%$ de estas estaciones presentó como componente principal la partícula limo con porcentajes entre $30,62 \%$ y $65,93 \%$ y el $48 \%$ de las estaciones restantes, la partícula arcilla, con porcentajes entre $40,86 \%$ y $73,88 \%$. Las estaciones con dominancia del $100 \%$ de fango se localizaron en el sector central del área relevada.
Tabla 3

Composición granulométrica (\%) y profundidad, de cada estación de muestreo en el sector sur del Golfo San Jorge

Granulometric composition (\%) and depth of each sampling station in the south sector of the San Jorge Gulf

\begin{tabular}{|c|c|c|c|c|}
\hline $\begin{array}{c}\text { Estación } \\
\text { General } \\
\end{array}$ & $\begin{array}{c}\text { Arenas } \\
\% \\
\end{array}$ & $\begin{array}{c}\text { Limo } \\
\% \\
\end{array}$ & $\begin{array}{c}\text { Arcilla } \\
\% \\
\end{array}$ & $\begin{array}{c}\text { Prof. } \\
\text { m }\end{array}$ \\
\hline 98 & - & 50,71 & 49,30 & 68 \\
\hline 99 & - & 51,62 & 48,38 & 58 \\
\hline 100 & - & 58,40 & 41,60 & 53 \\
\hline 101 & - & 49,04 & 50,96 & 51 \\
\hline 102 & - & 46,67 & 53,33 & 58 \\
\hline 103 & - & 55,93 & 44,07 & 43 \\
\hline 104 & 27,32 & 62,53 & 10,15 & 31 \\
\hline 106 & 62,08 & 27,20 & 10,72 & 31 \\
\hline 107 & 5,79 & 64,06 & 30,15 & 49 \\
\hline 109 & 5,79 & 65,93 & 28,28 & 70 \\
\hline 110 & 23,29 & 55,34 & 21,37 & 74 \\
\hline 111 & 37,27 & 41,84 & 20,89 & 48 \\
\hline 112 & 78,07 & 9,68 & 11,16 & 55 \\
\hline 114 & 45,76 & 33,51 & 20,73 & 86 \\
\hline 116 & 64,65 & 15,23 & 20,12 & 77 \\
\hline 117 & 43,11 & 35,59 & 21,30 & 84 \\
\hline 118 & 41,92 & 34,88 & 23,20 & 76 \\
\hline 119 & 49,41 & 34,59 & 16,00 & 76 \\
\hline 120 & 32,22 & 40,96 & 26,82 & 76 \\
\hline 122 & - & 46,00 & 54,00 & 66 \\
\hline 123 & - & 34,15 & 65,85 & 73 \\
\hline 124 & - & 41,12 & 58,88 & 64 \\
\hline 125 & - & 37,40 & 62,60 & 65 \\
\hline 126 & 1,23 & 45,66 & 53,11 & 56 \\
\hline 127 & 1,02 & 50,44 & 48,54 & 71 \\
\hline 129 & - & 40,71 & 59,29 & 71 \\
\hline 130 & 1,51 & 43,50 & 54,89 & 64 \\
\hline 131 & - & 26,12 & 73,88 & 79 \\
\hline 132 & - & 34,48 & 65,52 & 77 \\
\hline 133 & - & 38,30 & 61,70 & 79 \\
\hline 134 & - & 31,30 & 68,70 & 87 \\
\hline 135 & - & 29,67 & 70,33 & 85 \\
\hline 138 & - & 31,95 & 68,05 & 80 \\
\hline 140 & - & 29,70 & 70,30 & 80 \\
\hline 142 & - & 39,20 & 60,80 & 81 \\
\hline 144 & 49,52 & 30,62 & 19,86 & 66 \\
\hline 147 & 2,58 & 45,43 & 51,99 & 89 \\
\hline 148 & 15,11 & 44,25 & 40,64 & 86 \\
\hline 149 & 28,84 & 35,60 & 35,56 & 76 \\
\hline 150 & 17,93 & 37,04 & 45,03 & 73 \\
\hline 151 & 5,88 & 58,37 & 35,75 & 82 \\
\hline 152 & - & 48,80 & 51,20 & 81 \\
\hline 153 & 8,38 & 37,78 & 53,84 & 76 \\
\hline 155 & 5,03 & 55,77 & 39,20 & 56 \\
\hline 169 & 33,79 & 50,29 & 15,92 & 91 \\
\hline 170 & 34,91 & 24,23 & 40,86 & 91 \\
\hline
\end{tabular}



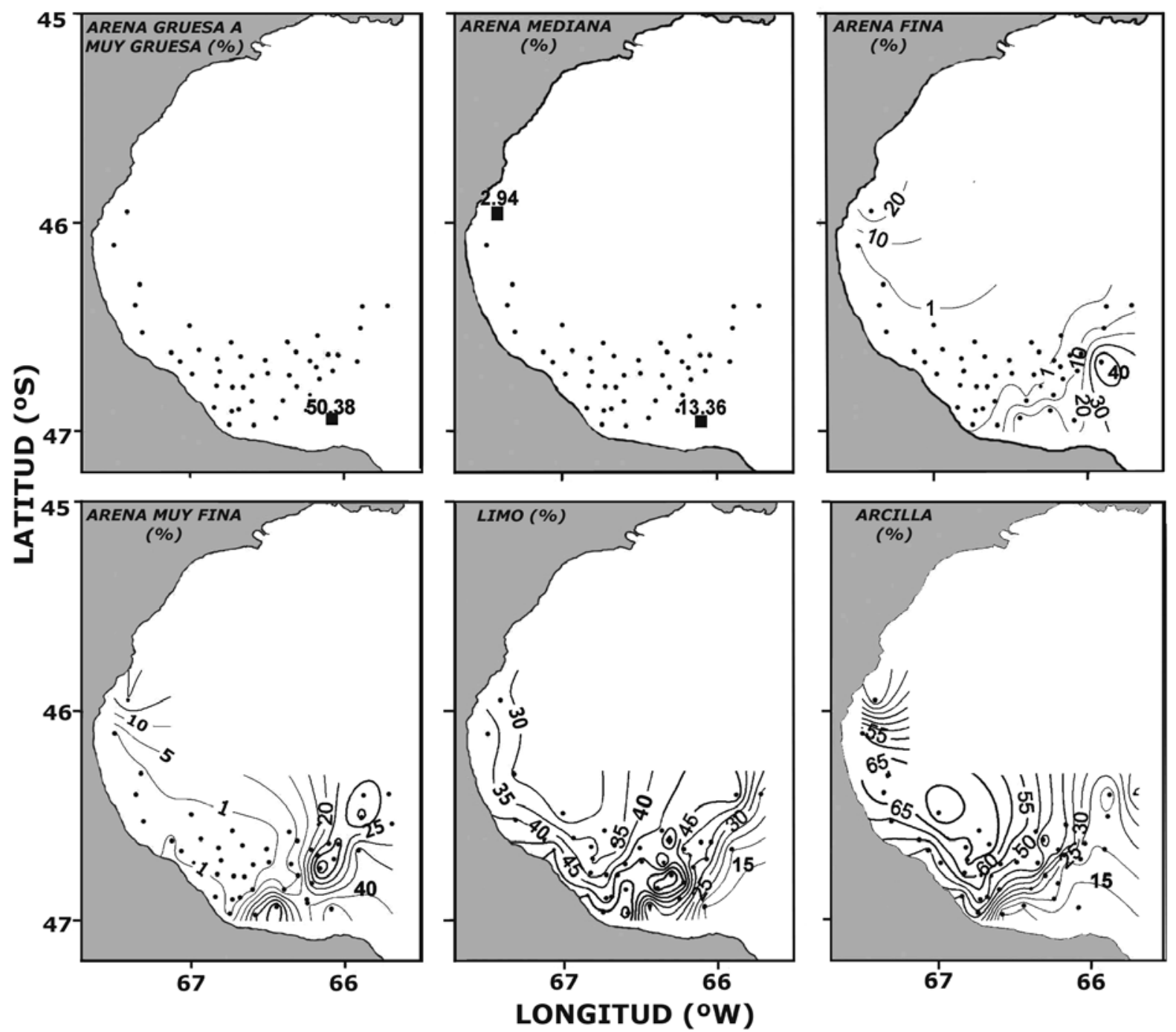

Figura 3

Distribución de las fracciones granulométricas de los sedimentos superficiales en el sector sur del Golfo San Jorge

Distribution of the granulometric fractions of the surficial sediments in the south sector of the San Jorge Gulf

\section{Caracterización química de los sedimentos}

\section{Materia orgánica total (MOT)}

Los valores determinados para esta variable en los sedimentos del sur del Golfo San Jorge fluctuaron entre $0,78 \%$ y $11,85 \%$ (Tablas 1 y 2). La distribución horizontal de la concentración de MOT presentó un gradiente positivo desde el extremo sudeste del golfo $(2,01 \%)$ hacia la zona costera oeste del área de estudio donde se registraron valores entre $8,56 \%$ y $9,38 \%$.

\section{Carbono orgánico total (COT)}

La concentración de COT en los sedimentos del área de estudio (Tablas 1 y 2) fluctuó entre $0,34 \%$ y $1,53 \%$, con un valor medio de $0,97 \%$. La distribución horizontal de esta variable (Fig. 4) presentó un aumento de los valores desde el extremo sudeste del golfo hacia la zona costera oeste del área de estudio donde se observó valores cercanos a $1,40 \%$. 


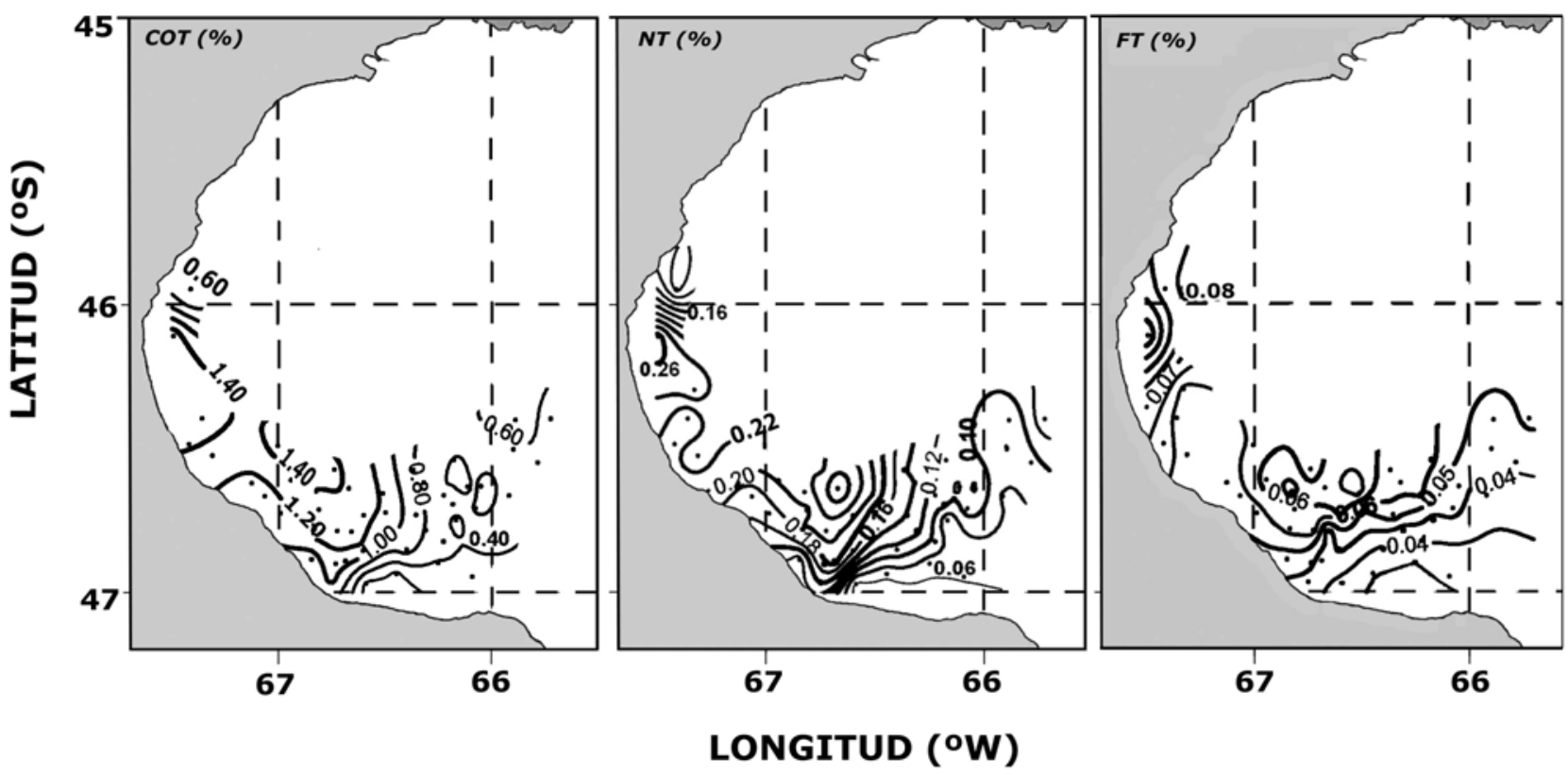

Figura 4

Distribución horizontal del carbono orgánico total COT (\%), nitrógeno total NT (\%) y fósforo total FT (\%) de los sedimentos superficiales en el sector sur del Golfo San Jorge

Horizontal distribution of the total organic carbon COT (\%), total nitrogen NT (\%) and total phosphorous FT (\%) of the surficial sediments in the south sector of the San Jorge Gulf

\section{Nitrógeno total (NT)}

La concentración de NT fluctuó entre $0,05 \%$ y $0,27 \%$ (Tablas 1 y 2). La distribución horizontal de esta variable (Fig. 4) presentó un aumento de los valores desde el sector sur y sudeste del golfo (con valores de $0,06 \%$ ) hacia la zona costera oeste del área de estudio (valores comprendidos entre $0,20 \%$ y $0,26 \%$ ). El valor máximo se registró en la estación 42 , ubicada en el oeste del golfo, próxima a la cuidad de Comodoro Rivadavia.

\section{Relación C:N (C:N)}

En los sedimentos superficiales del sur del Golfo San Jorge, los valores de la relación C: $\mathrm{N}$ variaron entre 4,73 y 8,54 , siendo el valor medio de 6,68 (Tablas 1 y 2 ). La distribución horizontal de esta variable no definió un claro patrón. Los valores de la relación C:N superiores a 7 se localizaron en el sector externo del golfo mientras que los inferiores a 7 en el sector costero oeste del golfo.

\section{Fósforo total (FT)}

La concentración de FT en los sedimentos del área de estudio (Tablas 1 y 2) fluctuó entre $0,03 \%$ y $0,09 \%$, con un valor medio de $0,05 \%$. La distribución horizontal de esta variable (Fig. 4) presentó un aumento de sus valores desde la zona costera sur del golfo (valores comprendidos entre $0,03 \%$ y $0,04 \%$ ) hacia la zona costera oeste del área de estudio (valores entre $0,05 \%$ y $0,09 \%$ ), respectivamente.

\section{Clorofila $a(\mathrm{Cl} a(\mathrm{~S}))$}

La concentración de clorofila $a$ en los sedimentos (Tablas 1 y 2) fluctuó entre $0,20 \mu \mathrm{g} \mathrm{g}^{-1}$ y $9,90 \mu \mathrm{g} \mathrm{g}^{-1}$, con un valor medio de $1,31 \mu \mathrm{g} \mathrm{g}^{-1}$. La distribución horizontal de esta variable no definió un claro patrón. En general, la distribución se observó muy uniforme, localizándose el registro máximo en la estación 140, ubicada en el sector costero oeste. 


\section{Feopigmentos (Feop (S))}

La concentración de feopigmentos en los sedimentos (Tablas 1 y 2) entre $1,74 \mu \mathrm{g} \mathrm{g}^{-1}$ y $43,19 \mu \mathrm{g} \mathrm{g}^{-1}$, con un valor medio de $11,98 \mu \mathrm{g} \mathrm{g}^{-1}$. La distribución horizontal de esta variable presentó un aumento de sus valores desde el extremo sudeste del golfo (valores comprendidos entre $1,74 \mu \mathrm{g} \mathrm{g}^{-1}$ y $3,05 \mu \mathrm{g} \mathrm{g}^{-1}$ ) hacia el área costera oeste, alcanzando allí valores superiores a 43,00 $\mu \mathrm{g} \mathrm{g}^{-1}$.

\section{Clasificación de las variables ambientales}

Los análisis de agrupamiento de las 27 variables físicoquímicas para las estaciones analizadas (Fig. 5) revelaron la existencia de dos agrupaciones importantes (grupos 1 y 2). El grupo 1 está caracterizado por las variables sedimentológicas arena gruesa a arena muy fina ( $\Phi 0$ a $\Phi 3,5)$, limo grueso $(\Phi 4)$ y relación C:N así como la temperatura, la concentración de oxígeno disuelto y la concentración de clorofila $a$ en agua de fondo. El grupo 2 lo definen las variables limo mediano a arcilla muy fina ( $\Phi 5$ a $\Phi 10)$, la concentración de MOT, COT, NT, FT, clorofila a y feopigmentos en sedimentos, la salinidad del agua de fondo y la profundidad de las estaciones. La clasificación de las estaciones se presenta gráficamente en la Fig. 6.

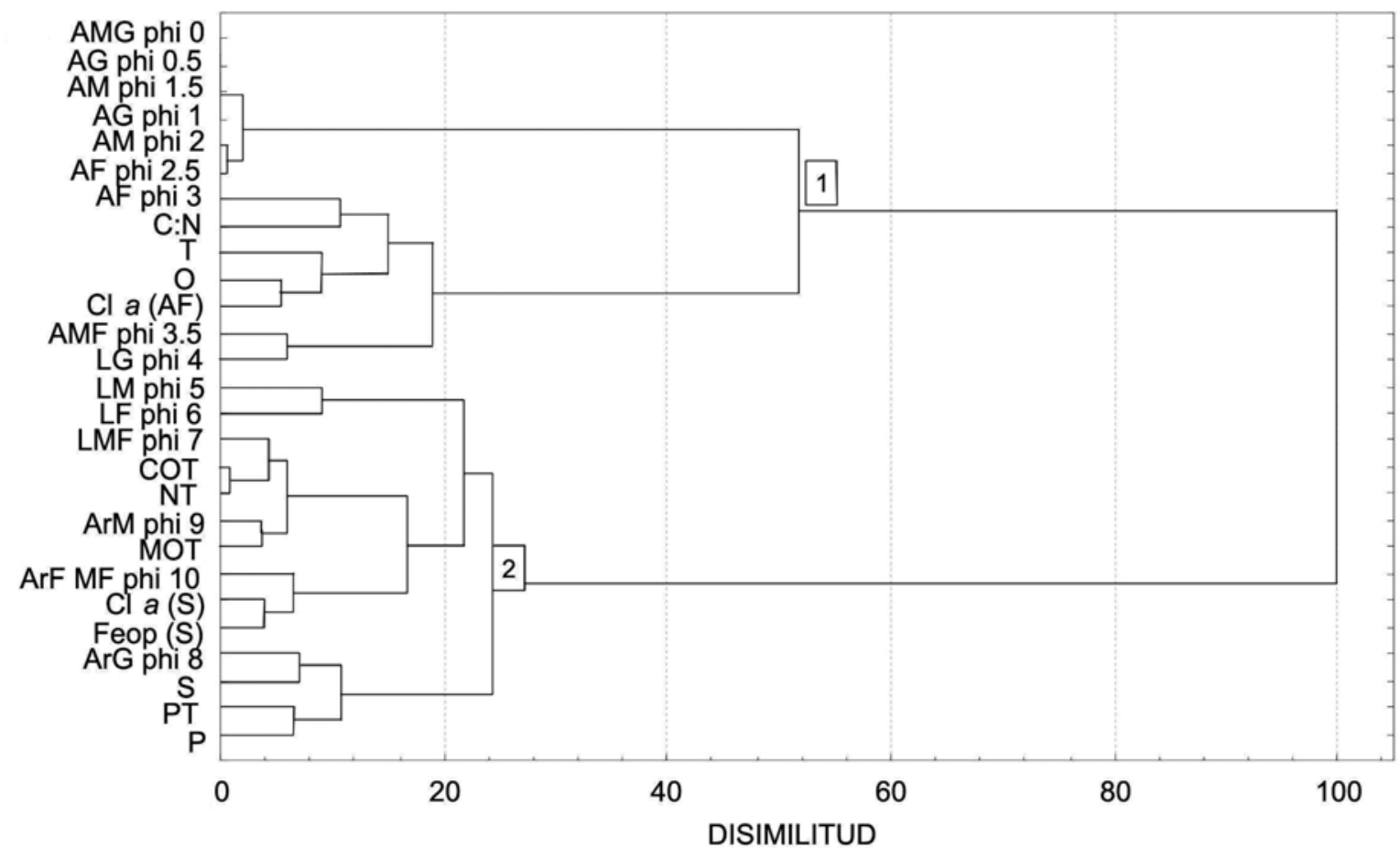

Figura 5

Análisis de agrupamiento entre las variables físico-químicas

Cluster analysis among physico-chemical variables 


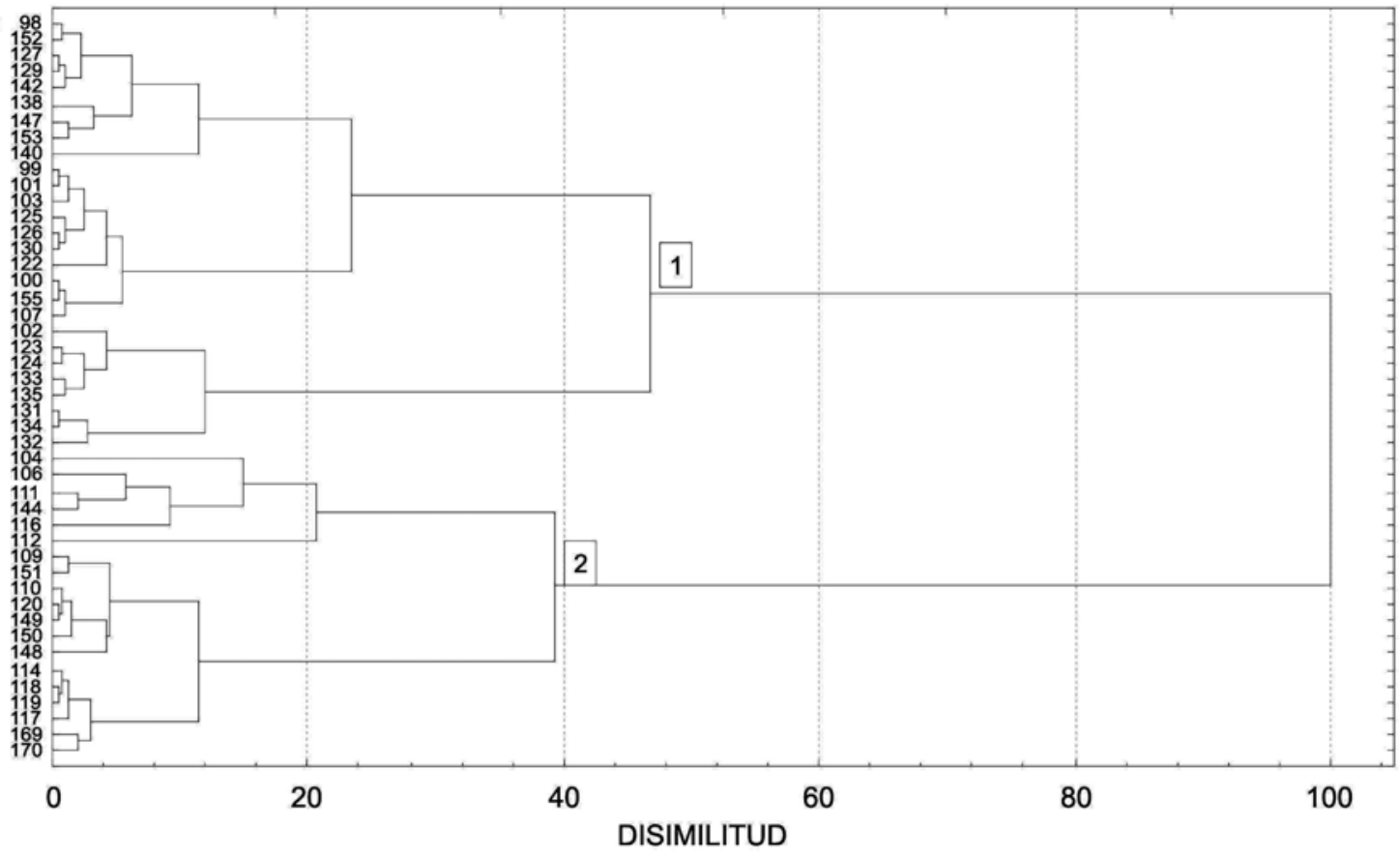

Figura 6

Análisis de agrupamiento entre estaciones de muestreo

Cluster analysis among sampling stations

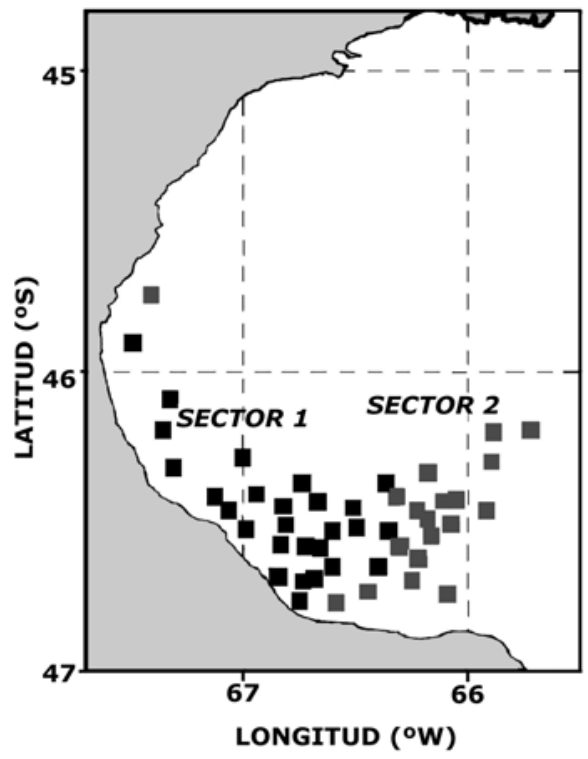

Figura 7

Análisis de agrupamiento entre sectores definidos en el área de estudio

Cluster analysis among defined sectors in the studied area
Se observaron dos agrupaciones. Por un lado, las estaciones ubicadas en la zona interna, costera oeste del área de estudio (grupo 1) y por otro, las estaciones situadas en el sector externo del área y la estación costera ubicada frente a la ciudad de Comodoro Rivadavia (grupo 2) (Fig. 7). En la Tabla 4 se presenta la estadística descriptiva de las variables físicoquímicas de los sedimentos y del agua de fondo, de los sectores definidos.

\section{Discusión}

Los valores de temperatura determinados en el agua de fondo para el sur del Golfo San Jorge son superiores a los registrados por Fernández et al. (2006), los que fluctuaron entre $5,83{ }^{\circ} \mathrm{C}$ y $8,18{ }^{\circ} \mathrm{C}$. Este incremento de la temperatura concuerda con la anomalía promedio del agua superficial del Golfo San Jorge detectada para la serie de tiempo enero de 1982 - julio de 2005. Esta anomalía se desvía de la media, particularmente en forma sostenida a partir de 1997, siendo los valores superiores a los valores promedio (Proyecto Langostino 2005). 
Tabla 4

Estadística descriptiva de las variables físico-químicas de los sedimentos y del agua de fondo, en los sectores definidos en el sector sur del Golfo San Jorge

Descriptive statistic of sediment and bottom water physico-chemical variables in the defined sectors in the south sector of the San Jorge Gulf

\begin{tabular}{|c|c|c|c|c|c|c|c|c|}
\hline \multirow[b]{2}{*}{ Variables } & \multicolumn{4}{|c|}{ Sector 1} & \multicolumn{4}{|c|}{ Sector 2} \\
\hline & $\begin{array}{c}\text { valor } \\
\text { mínimo } \\
\end{array}$ & $\begin{array}{c}\text { valor } \\
\text { máximo }\end{array}$ & $\begin{array}{c}\text { valor } \\
\text { promedio }\end{array}$ & $\begin{array}{c}\text { desviación } \\
\text { estándar }\end{array}$ & $\begin{array}{c}\text { valor } \\
\text { mínimo }\end{array}$ & $\begin{array}{c}\text { valor } \\
\text { máximo }\end{array}$ & $\begin{array}{c}\text { valor } \\
\text { promedio }\end{array}$ & $\begin{array}{c}\text { desviación } \\
\text { estándar }\end{array}$ \\
\hline Profundidad (m) & 43,00 & 89,00 & 68,89 & 12,65 & 31,00 & 91,00 & 71,00 & 17,80 \\
\hline Arena muy gruesa $(\Phi 0)(\%)$ & 0 & 0 & 0 & 0 & 0 & 44,95 & 2,37 & 10,31 \\
\hline Arena gruesa $(\Phi 0,5)(\%)$ & 0 & 0 & 0 & 0 & 0 & 3,04 & 0,16 & 0,70 \\
\hline Arena gruesa ( $\Phi$ 1) (\%) & 0 & 0 & 0 & 0 & 0 & 2,39 & 0,13 & 0,55 \\
\hline Arena gruesa a muy gruesa $(\%)$ & 0 & 0 & 0 & 0 & 0 & 50,38 & 2,65 & 11,56 \\
\hline Arena mediana $(\Phi 1,5)(\%)$ & 0 & 0 & 0 & 0 & 0 & 3,26 & 0,17 & 0,75 \\
\hline Arena mediana (Ф 2) $(\%)$ & 0 & 0 & 0 & 0 & 0 & 10,10 & 0,69 & 2,38 \\
\hline Arena mediana $(\%)$ & 0 & 0 & 0 & 0 & 0 & 13,36 & 0,86 & 3,10 \\
\hline Arena fina $(\Phi 2,5)(\%)$ & 0 & 0 & 0 & 0 & 0 & 12,05 & 1,34 & 2,90 \\
\hline Arena fina ( $\Phi$ 3) $(\%)$ & 0 & 1,21 & 0,16 & 0,31 & 0,47 & 48,89 & 9,75 & 12,64 \\
\hline Arena fina $(\%)$ & 0 & 1,21 & 0,16 & 0,31 & 0,47 & 51,92 & 11,02 & 13,37 \\
\hline Arena muy fina $(\Phi 3,5)(\%)$ & 0 & 7,45 & 0,79 & 1,83 & 1,09 & 40,29 & 22,21 & 11,79 \\
\hline Total arena $(\%)$ & 0 & 8,38 & 0,95 & 2,12 & 5,79 & 79,16 & 36,73 & 19,42 \\
\hline Limo grueso (Ф 4) (\%) & 0,92 & 23,38 & 9,11 & 5,85 & 2,75 & 47,31 & 19,74 & 9,17 \\
\hline Limo medio ( $\Phi$ 5) (\%) & 0,03 & 19,59 & 6,84 & 4,86 & 0,26 & 17,67 & 6,04 & 4,61 \\
\hline Limo fino $(\Phi 6)(\%)$ & 1,69 & 31,65 & 12,61 & 5,89 & 1,19 & 14,92 & 7,01 & 3,81 \\
\hline Limo muy fino (Ф 7) (\%) & 2,10 & 20,29 & 14,55 & 3,85 & 2,27 & 12,23 & 6,02 & 2,51 \\
\hline Total limo (\%) & 26,13 & 64,06 & 43,04 & 9,55 & 9,68 & 65,93 & 38,83 & 14,97 \\
\hline Arcilla gruesa $(\Phi 8)(\%)$ & 0,88 & 61,25 & 22,04 & 15,32 & 2,84 & 29,25 & 9,13 & 6,48 \\
\hline Arcilla media (Ф 9) (\%) & 4,10 & 18,35 & 10,74 & 4,11 & 0,97 & 9,66 & 4,91 & 2,55 \\
\hline Arc. fina a muy fina $(\Phi 10)(\%)$ & 6,43 & 58,57 & 23,16 & 12,26 & 2,00 & 24,37 & 10,40 & 6,18 \\
\hline Total arcilla $(\%)$ & 30,15 & 73,88 & 55,48 & 10,63 & 10,15 & 45,03 & 25,36 & 11,13 \\
\hline Materia orgánica total (\%) & 0,78 & 11,85 & 8,37 & 2,21 & 2,01 & 6,34 & 3,99 & 1,04 \\
\hline Carbono orgánico total (\%) & 0,79 & 1,53 & 1,22 & 0,21 & 0,34 & 0,95 & 0,62 & 0,17 \\
\hline Nitrógeno total (\%) & 0,11 & 0,27 & 0,19 & 0,04 & 0,05 & 0,14 & 0,09 & 0,03 \\
\hline Fósforo total (\%) & 0,04 & 0,09 & 0,05 & 0,01 & 0,03 & 0,07 & 0,05 & 0,01 \\
\hline Relación C:N & 5,27 & 8,54 & 6,69 & 0,83 & 4,73 & 8,51 & 6,67 & 1,36 \\
\hline Clorofila $a(\mathrm{~S})\left(\mu \mathrm{g} \mathrm{g}^{-1}\right)$ & 0,49 & 9,90 & 1,68 & 1,78 & 0,20 & 1,77 & 0,79 & 0,44 \\
\hline Feopigmentos $(\mathrm{S})\left(\mu \mathrm{g} \mathrm{g}^{-1}\right)$ & 4,53 & 43,19 & 16,01 & 7,95 & 1,74 & 14,29 & 6,25 & 3,39 \\
\hline Temperatura $\left({ }^{\circ} \mathrm{C}\right)$ & 8,39 & 9,76 & 9,05 & 0,36 & 8,51 & 9,68 & 9,15 & 0,35 \\
\hline Salinidad (ups) & 33,31 & 33,56 & 33,41 & 0,07 & 33,13 & 33,51 & 33,37 & 0,08 \\
\hline Densidad $\left(\sigma_{t}\right)$ & 25,67 & 26,08 & 25,87 & 0,12 & 25,72 & 26,02 & 25,82 & 0,09 \\
\hline Concentración Oxígeno $\left(\mathrm{O}_{2} \mathrm{~L}^{-1}\right)$ & 1,30 & 6,20 & 4,71 & 1,28 & 2,90 & 6,27 & 4,95 & 1,00 \\
\hline Clorofila $a(\mathrm{AF})\left(\mathrm{mg} \mathrm{m}^{-3}\right)$ & 0,02 & 0,91 & 0,40 & 0,25 & 0,03 & 0,77 & 0,48 & 0,24 \\
\hline
\end{tabular}


Los valores de salinidad registrados en el agua de fondo son similares a los obtenidos por otros autores (Akselman 1996, Fernández et al. 2005, 2006). Estos valores indican la existencia de dos masas de aguas: 1) las denominadas aguas de plataforma $(33,2<\mathrm{S}>33,8$ ups) y 2) las aguas costeras ( $\mathrm{S}<33,2$ ups). Las aguas costeras se originan como consecuencia de la mezcla de agua subantártica procedente de la Corriente del Cabo de Hornos con agua que entra al Atlántico por el Estrecho de Magallanes, diluidas por aportes continentales (Brandhorst \& Castello 1971, Bianchi et al. 1982). Las aguas costeras fluyen pegadas a la costa de la Provincia de Santa Cruz, bifurcándose su flujo a la altura del cabo Tres Puntas en dos ramas principales. Una de las ramas ingresa al golfo por su extremo sudeste y ejerce una notable influencia en esa región durante todo el año. La otra rama se aleja de la costa en dirección N-NE, afectando en toda su extensión a la región del umbral del golfo (Fernández et al. 2005).

Los valores calculados de densidad fueron inferiores a los registrados en el invierno de 2001 por Fernández et al. (2006), cuyo valor medio fue de 26,297. La distribución horizontal y vertical de la variable refleja en el sector sur hacia el sector central del umbral del golfo un frente termohalino definido por el ingreso de las aguas costeras tal como lo definieran Glorioso \& Flather (1995).

Los valores de concentración de oxígeno disuelto para el agua de fondo se observaron inferiores a los registrados durante el invierno de 2001 (Fernández et al. 2006) cuyo valor medio fue de $6,73 \mathrm{~mL} \mathrm{O}_{2} \mathrm{~L}^{-1}$. Sin embargo, los mismos se encuentran dentro de los valores normales citados para ambientes de plataforma continental oxigenados con valores de oxígeno disuelto superiores a $3 \mathrm{~mL} \mathrm{O}_{2} \mathrm{~L}^{-1}$ (Bernd \& Simoneit 1978); a excepción de cuatro registros localizados en el sector central del sur del golfo que indican condiciones de hipoxia $\left(3 \mathrm{~mL} \mathrm{O}_{2} \mathrm{~L}^{-1}-0,5 \mathrm{~mL} \mathrm{O}_{2} \mathrm{~L}^{-1}\right.$ ) (Damaison \& Moore 1980).

Durante el otoño-invierno de 2003, los valores promedio de clorofila $a$ en la capa de fondo fueron inferiores a los registrados por Fernández et al. (2006), cuyo valor medio fue de $1,63 \mathrm{mg} \mathrm{m}^{-3}$. Las variaciones estacionales de biomasa fitoplanctónica en el Golfo San Jorge, presentan el característico ciclo bimodal de aguas templadas, con un máximo principal durante la primavera y otro secundario durante el otoño, observándose durante la etapa invernal una homogeneidad en la distribución vertical y horizontal de los valores de clorofila $a$, hecho observado durante el presente estudio.

La cartografía sedimentaria en el otoño-invierno de 2003 respecto a la confeccionada previamente en primavera de 1999 (Fernández et al. 2003) presentó un mayor porcentaje de granulometría fina, posiblemente consecuencia de la dinámica del sector así como de la actividad pesquera que provoca la resuspensión de los sedimentos (Bertuche et al. 2000, Roux 2000). La distribución de los sedimentos en el golfo permite diferenciar: 1) la zona interna con profundidades mayores a $53 \mathrm{~m}$, con sedimentos fangosos con alto contenido en limos y arcillas y 2) la zona litoral y el sudeste del golfo con profundidades comprendidas entre los 21 y $53 \mathrm{~m}$, con un sustrato constituido por arenas. En esta última zona se diferencia en el extremo sur del golfo, una distribución de elementos gruesos, principalmente grava y arenas. Este comportamiento espacial coincide con lo observado por Roux et al. (1995) y Fernández et al. (2003).

Para el sur del Golfo San Jorge, los valores obtenidos de materia orgánica total coinciden de manera general con las concentraciones señaladas como normales para sedimentos marinos (entre 0,1\% 10\%) (Establier et al. 1984).

Los valores de carbono orgánico total concuerdan con los encontrados por Premuzic et al. (1982) para los sedimentos de la plataforma continental argentina (valores entre $0,25 \%$ y $2,00 \%$ ).

Los valores de nitrógeno total superan levemente el intervalo de valores citado por Premuzic et al. (1982) para la plataforma continental argentina $(0,06 \%$ a $0,20 \%)$.

Los valores de la relación $\mathrm{C}: \mathrm{N}$ registrados sugieren según lo señalado por Parsons et al. (1977) y Sargent et al. (1983) que la materia orgánica de los sedimentos estaría fundamentalmente constituida por material de origen planctónico autóctono, derivado de la producción primaria y secundaria del sistema y de alto valor nutricional.

Los valores de fósforo total fueron inferiores a los registrados en ambientes caracterizados por procesos de eutroficación y contaminación (Rudolph et al. 1984) como así también a los de ambientes influenciados por el aporte de agua proveniente del lavado del terreno con importante carga de fosforados por actividad 
agrícola (El Sabrouti et al. 1997). Los valores fueron semejantes a los registrados en la Bahía de Pomerania, Mar Báltico, sistema sin indicios de contaminación y donde se observa un florecimiento fitoplanctónico primaveral y dos secundarios, uno durante el verano y otro en el otoño (Burska et al. 1999).

Los valores de clorofila $a$ registrados en el sedimento durante el presente estudio fueron inferiores a los registrados en el invierno de 2001 (Fernández et al. 2006) cuyo valor medio fue de $1,54 \mu \mathrm{g} \mathrm{g}^{-1}$; mientras que el valor medio de feopigmentos fue también, inferior al observado en el período antes mencionado por Fernández et al. (2006) cuyo valor medio fue de $20,50 \mu \mathrm{g} \mathrm{g}^{-1}$.

En cuanto a los sectores definidos mediante la técnica estadística aplicada, el sector 1 corresponde a un Ambiente Deposicional y el sector 2, a un Ambiente Transicional, correspondiendo a lo observado por Fernández et al. (2003, 2005, 2006). Estos autores definieron en estudios que contemplaron al golfo en su totalidad, empleando técnicas estadísticas basadas en la distribución espacial de las fracciones granulométricas, la composición química de los sedimentos superficiales y la composición físico - química del agua de fondo así como de sus variaciones estacionales, tres sectores bien diferenciados, el Ambiente Deposicional (centro del golfo), el Ambiente de Flujo o Erosivo (sectores próximos a los cabos Dos Bahías y Tres Puntas) y el Ambiente Transicional (sectores costeros y sudeste del golfo).

Coincidiendo con los autores antes mencionados, durante el presente estudio el sector 1 correspondiente al Ambiente Deposicional, desde el punto de vista hidrográfico, presenta un ciclo típico de aguas templadas. El sistema está caracterizado por condiciones hidrodinámicas bajas y estables que favorecen la depositación de elementos granulométricos finos provenientes de los ambientes menos profundos del golfo y de material orgánico suspendido en la columna de agua. Los valores registrados de las variables químicas de los sedimentos durante nuestro estudio, se corresponden con la ruptura de la termoclina durante este período, lo que permite que la materia orgánica particulada alcance el fondo. Este acople entre el sistema pelágico y el bentos unido a la menor actividad biológica impuesta por la baja temperatura imperante, está de acuerdo con la mayor abundancia y calidad nutritiva de la materia orgánica asociada a dichos sedimentos durante el otoño-invierno (Fernández et al. 2003, 2005, 2006).
El sector 2, correspondiente al Ambiente Transicional presenta características intermedias entre el Ambientes Deposicional y el de Flujo o Erosivo. Este último, que no se encuentra representado durante nuestro estudio, dadas las características granulométricas tanto físicas como químicas de los sedimentos (granulometría gruesa y bajas concentraciones de material orgánico) se caracteriza por el flujo o movimiento continuo de agua que genera una elevada energía cinética y la erosión de las partículas granulométricas. Este flujo está dado por la acción de las corrientes de marea y principalmente en el extremo sur del golfo por el ingreso de las aguas costeras provenientes del Estrecho de Magallanes. En este sistema no se observa la formación de una termoclina dada la continua turbulencia del medio. Esto favorece la homogenización y suspensión de la materia orgánica particulada en la columna de agua dificultando la sedimentación de la misma, lo que concuerda con los bajos valores de concentración de componentes orgánicos registrados en los sedimentos. En consecuencia, el sector 2 presenta, en su parte más litoral, características del Ambiente de Flujo o Erosivo, tales como menor profundidad y elevado hidrodinamismo, y en su parte más profunda, características del Ambiente Deposicional tales como bajo hidrodinamismo y depositación. Estas particularidades hidrográficas permiten la formación de sistemas frontales (frentes de marea) de variabilidad espacial marcada. A la vez, este sector está bajo la influencia de las aguas costeras de origen magallánico que generan un frente termohalino de permanencia estacional. Los sedimentos del sector, constituidos por arenas muy finas, presentan concentraciones moderadas de MOT, COT, NT y feopigmentos, que pueden considerase concentraciones intermedias con respecto a las registradas para el Ambiente Deposicional y el Ambiente de Flujo o Erosivo (Fernández et al. 2003, 2005, 2006).

Esta caracterización del área de estudio, que se define como la de mayor concentración del recurso langostino en aguas patagónicas, es un aporte más al conocimiento del ecosistema del Golfo San Jorge y nos brinda nuevas herramientas, en beneficio del manejo de una de las pesquerías más importantes del Mar Argentino. Las variables analizadas en el presente trabajo serán relacionados con los índices de abundancia de la especie, tema que será desarrollado en futuras investigaciones. En diversas pesquerías de peneidos se han identificado correlaciones significativas entre la distribución de los organismos y ciertas variables ambientales. En general se observa una correlación significativa entre los índices de abundancia de las 
especies con dos características de los sedimentos: 1) el tamaño de las partículas, de importancia desde el punto de vista de protección y refugio y 2) el contenido de carbono orgánico, considerado una medida indirecta de la disponibilidad de alimento. Esta correlación ha sido observada en pesquerías de peneidos del Golfo de México (Williams 1958, Grady 1971), en la Bahía de Mobile, Alabama, USA (Loesch 1965), de la costa Sudanesa del Mar Rojo (Branford 1981), y del Golfo de Carpentaria, Australia (Somers 1987), entre otras.

\section{Agradecimientos}

Sinceros agradecimientos a los evaluadores por su importante aporte.

\section{Literatura citada}

Akselman R. 1996. Estudios ecológicos en el Golfo San Jorge y adyacencias (Atlántico sudoccidental). Distribución, abundancia y variación estacional del fitoplancton en relación a factores físico-químicos y a la dinámica hidrográfica. Tesis Doctoral, Universidad de Buenos Aires, Buenos Aires, Argentina. 234 pp.

Aubone A, S Bezzi, R Castrucci, C Dato, P Ibáñez, G Irusta, M Perez, M Renzi, B Santos, N Scarlato, M Simonazzi, L Tringalli \& F Villarino. 2000. Merluza (Merluccius hubbsi). En: Bezzi S, R Akselman \& E Boschi (eds), Síntesis del estado de las Pesquerías Marítimas Argentinas y de la Cuenca del Plata. Años 1997-1998, con la actualización de 1999: 29-39. INIDEP, Mar del Plata, Argentina.

Bernd R \& T Simoneit. 1978. The organic chemistry of marine sediments. En: Riley JP \& R Chester (eds), Chemical Oceanography, 7, $2^{\text {nd }}$ ed., pp. 234-311, Academic Press, London.

Bertuche D, C Fischbach, A Roux, M Fernández \& R Piñero. 2000. Langostino (Pleoticus muelleri). En: Bezzi S, R Akselman \& E Boschi (eds), Síntesis del estado de las Pesquerías Marítimas Argentinas y de la Cuenca del Plata. Años 1997-1998, con la actualización de 1999: 179-190. INIDEP, Mar del Plata, Argentina.

Bianchi A, M Masonneau \& R Oliviera. 1982. Análisis estadístico de las características T-S del sector austral de la plataforma continental argentina. Acta Oceanográfica Argentina 3: 93-118.
Brandhorst W \& J Castello. 1971. Evaluación de los recursos de anchoita (Engraulis anchoita) frente a la Argentina y Uruguay. Proyecto de Desarrollo Pesquero (FAO), Serie Informe Técnico 29: 1-63.

Branford JR. 1981. Sediment and the distribution of Penaeid shrimp in the Sudanese Red Sea. Estuarine, Coastal and Shelf Science 13: 349-355.

Buchanan JB. 1984. Sediment analysis. En: Holme NA \& AD Mc Intyre (eds), Methods for the study of marine benthos, pp. 41-65. Blackwell Scientific Publications, Oxford.

Burska D, L Frankowski \& J Bolalek. 1999. Temporal variability in the chemical composition of bottom sediments in the Pomeranian Bay (southern Baltic). Oceanologia 41 (3): 445-459.

Damaison G \& GT Moore. 1980. Anoxic environments and oil source bed genesis. Organic Geochemistry 2: 9-31.

El Sabrouti MA, KH Abaza \& BA Mahmoud. 1997. Organic matter, phosphorus and sulfur distribution in the surficial sediments of Manzalah lagoon, Egypt. Fresenius Environment Bulletin 6: 403-409.

Establier R, J Blasco, A Gómez \& D Escobar. 1984. Materia orgánica en los sedimentos de la Bahía de Cádiz y sus zonas de marismas y salinas. Investigación Pesquera 48 (2): $285-301$.

Fernández M. 2006. Características físico-químicas de los sedimentos del Golfo San Jorge y su relación con los organismos bentónicos del sector. Tesis Doctoral, Universidad Nacional de Mar del Plata, Argentina, 307 pp.

Fernández M \& D Bertuche. 1995. Relevamiento del estado de los sedimentos de los fondos de pesca de la pesquería de langostino patagónico. Año 1992 y 1993. Informe Técnico Interno DNI INIDEP No 5/95: 1-16.

Fernández M, A Roux, E Fernández, J Caló, A Marcos \& H Aldacur. 2003. Grain-size analysis of surficial sediments from Golfo San Jorge, Argentina. Journal of the Marine Biological Association of the United Kingdom 83: 1193-1197.

Fernández M, J Carreto, J Mora \& A Roux. 2005. Physico-chemical characterization of the benthic ambient of Golfo San Jorge, Argentina. Journal of the Marine Biological Association of the United Kingdom 85: 13171328 . 
Fernández M, J Mora, A Roux, D Cucchi Colleoni \& J Gasparoni. En prensa. A new contribution on spatial and seasonal variability of environmental conditions of the Golfo San Jorge, Argentina. Journal of the Marine Biological Association of the United Kingdom.

Glorioso P \& R Flather. 1997. The Patagonia shelf tides. Progress in Oceanography 40: 263-283.

Grady JR. 1971. The distribution of sediment properties and shrimp catch on two shrimping grounds on the continental shelf of Mexico. Proceedings of the Gulf and Caribbean Fisheries Institute 23: 39-48.

Loesch L. 1965. Distribution and growth of penaeid shrimp in Mobile Bay, Alabama. Publications of the Institute of Marine Science, 453 pp. Parker P \& BJ Copeland (eds). University of Texas. Port Aransas, Texas.

Parsons TR, M Takahashi \& B Hargreav. 1977. Biological Oceanographic Processes, 490 pp. $2^{\text {nd }}$ ed. Pergamon Press, Oxford.

Premuzic ET, C Bencovitz, J Gaffney \& J Walsh. 1982. The nature and distribution of organic matter in the surface sediments of world oceans and seas. Organic Geochemistry 4: 63-77.

Proyecto Langostino. 2005. Informe presentado a la Consultora Serman \& Asociados S.A, 162 pp. INIDEP, Mar del Plata, Argentina.

Roux A. 2000. Evaluación del impacto pesquero a través del análisis de la fauna bentónica acompañante en la pesquería de langostino (Pleoticus muelleri) del Golfo
San Jorge y litoral de Chubut, Argentina. Frente Marítimo 18: 143-149.

Roux A \& M Fernández. 1997. Caracterización de los fondos de pesca del langostino Pleoticus muelleri en el Golfo San Jorge y litoral de la provincia de Chubut. Argentina. Serie Informe Técnico Interno INIDEP $\mathrm{N}^{\circ} 13$, $28 \mathrm{pp}$.

Roux A, M Fernández \& C Bremec. 1995. Preliminary survey of the benthic communities of patagonian shrimp fishing grounds in San Jorge Gulf (Argentina). Ciencias Marinas 21 (3): 295-310.

Rudolph A, R Ahumada \& S Hernández. 1984. Distribución de la materia orgánica, carbono orgánico, nitrógeno orgánico y fósforo total en los sedimentos recientes de la Bahía de Concepción, Chile. Biología Pesquera 13: 71-82.

Sargent J, C Hopkins, J Seiring \& A Youngson. 1983. Partial characterization of organic material in surface sediments from Balsfjorden, northern Norway, in relation to its origin and nutritional value for sediment-ingesting animals. Marine Biology 76: 87-94.

Somers I. 1987. Sediment type as a factor in the distribution of the commercial prawn species of the western Gulf of Carpentaria, Australia. Australian Journal of Marine and Freshwater Research 38:133-149.

Strickland, JHD \& TR Parsons. 1965. A manual of sea water analysis. Bulletin of the Fisheries Research Board of Canada. 125, 212 pp.

Recibido el 17 de agosto de 2006 y aceptado el 3 de julio de 2007 